\title{
Transport in an Idealized Three-Gyre System with Application to the Adriatic Sea
}

\author{
IRINA I. RYPINA \\ Woods Hole Oceanographic Institution, Woods Hole, Massachusetts \\ MiCHAEL G. BROWN \\ RSMAS, University of Miami, Miami, Florida \\ HUSEYIN KOÇAK \\ Departments of Computer Science and Mathematics, University of Miami, Coral Gables, Florida
}

(Manuscript received 14 January 2008, in final form 21 August 2008)

\begin{abstract}
Motivated by observations of surface drifters in the Adriatic Sea, transport in a three-gyre system is studied with the aid of dynamical systems techniques. Particular attention is paid to the issue of intergyre transport. The velocity field is assumed to be two-dimensional and incompressible and composed of a steady three-gyre background flow on which a time-dependent perturbation is superimposed. Two systems of this type are considered: 1) an observationally motivated, analytically prescribed model consisting of a steady background on which a multiperiodic time-dependent perturbation is superimposed, and 2) an observationally based model of the Adriatic Sea consisting of the mean surface circulation derived from surface drifter trajectories on which a time-dependent altimetry-based perturbation velocity field is superimposed. It is shown that for a small perturbation to the steady three-gyre background, two of the gyres exchange no fluid with the third gyre. When the perturbation strength exceeds a certain threshold, transport between all three gyres occurs. This behavior is described theoretically, illustrated using the analytic model and shown to be consistent with the observationally based model of the Adriatic. The relevance of the work presented to more complicated multiple-gyre problems is discussed.
\end{abstract}

\section{Introduction}

Figure 1 [see also Fig. 1b in Poulain (2001)] shows a large ensemble of surface drifter trajectories in the Adriatic Sea. The trajectories shown cover the period from 1 August 1990 to 31 July 1999. These and other similar measurements have motivated a number of studies of the Lagrangian description of near-surface fluid motion in the Adriatic Sea (Orlic et al. 1992; Falco et al. 2000; Maurizi et al. 2004; Castellari et al. 2001; Artegiani et al. 1997a,b; Poulain 1999; Cushman-Roisin et al. 2001; Poulain 2001; Lacorata et al. 2001; Haza et al. 2007b,a). Figure 1 gives insight into the mean surface circulation, its variability, and the complexity of the associated Lagrangian motion. A striking feature is

Corresponding author address: Dr. Irina I. Rypina, Physical Oceanography Department, Woods Hole Oceanographic Institution, 360 Woods Hole Road, Woods Hole, MA 02543.

E-mail: irypina@whoi.edu the robustness of the background multiple-gyre flow pattern. Neglecting the small northernmost gyre in the Adriatic Basin (Fig. 1, left), there appear to be three dominant gyres. The average circulation in all three gyres is cyclonic. Drifters sometimes get trapped for a long time in one of the gyres, but many examples of drifters moving from one gyre to another can also be seen. These observations suggest the following question, which is the central theme of this paper: What controls intergyre transport in a three-gyre system?

The Lagrangian equations of motion are

$$
\frac{\mathrm{d} \mathbf{x}}{\mathrm{d} t}=\mathbf{u}(\mathbf{x}, t)
$$

where $\mathbf{u}(\mathbf{x}, t)$ is the Eulerian velocity field. Most of the previous work on the Lagrangian description of fluid motion in the Adriatic Sea has adopted a stochastic framework (Castellari et al. 2001; Maurizi et al. 2004; Poulain 2001; Lacorata et al. 2001; Haza et al. 2007a). 


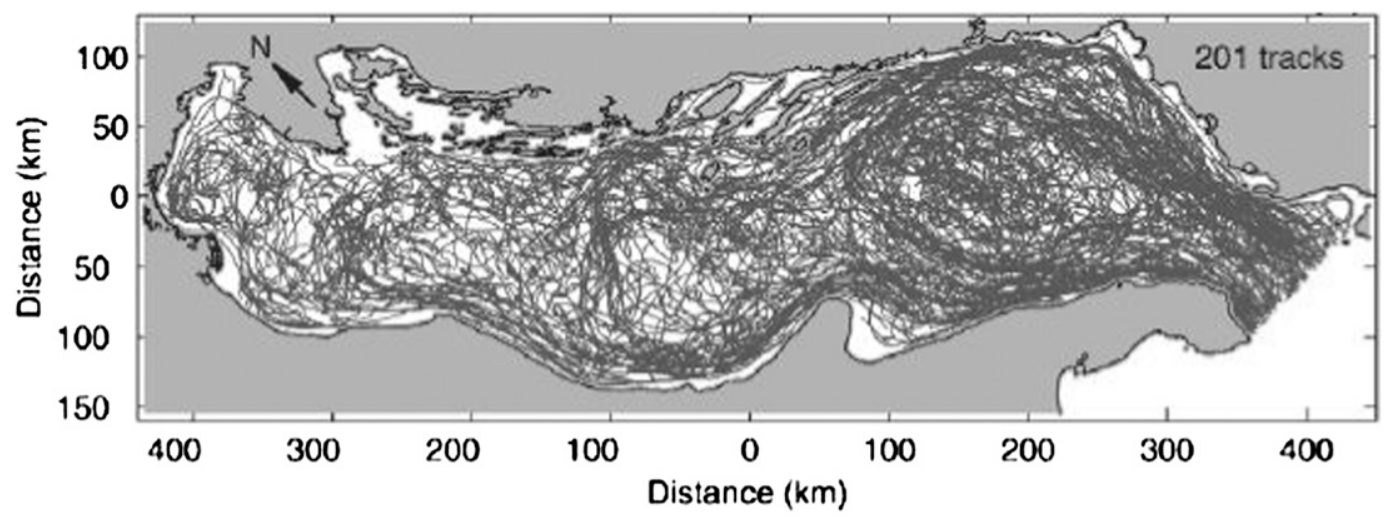

FIG. 1. Tracks of 201 surface drifters in the Adriatic Sea between 1 August 1990 and 31 July 1999. Figure adapted from Poulain (2001).

This involves invoking the assumption that there is a separation of scales between deterministic and stochastic components of velocity field $\mathbf{u}=\mathbf{U}+\mathbf{u}^{\prime}$, which leads to an advection-diffusion or Fokker-Planck equation to describe transport. Each fluid particle trajectory is then controlled by two different processes: 1) advection by the large-scale velocity field $\mathbf{U}$ and 2) turbulent transport by the small-scale stochastic perturbation field $\mathbf{u}^{\prime}$, whose influence is parameterized by an effective diffusivity.

An alternative approach to the study of fluid transport processes and Lagrangian fluid dynamics is to apply results associated with dynamical systems theory. Equation (1) defines a dynamical system. We consider here the special case of a two-dimensional $\mathbf{x}=(x, y)$ and incompressible velocity field. For this class of problems one can introduce a streamfunction $\psi(x, y, t)$ and the Lagrangian equations of motion are

$$
\frac{d x}{d t}=-\frac{\partial \psi}{\partial y}, \frac{d y}{d t}=\frac{\partial \psi}{\partial x}
$$

It is well known that these equations have Hamiltonian form with the streamfunction playing the role of the Hamiltonian. The Hamiltonian structure of Eqs. (2) will be discussed extensively in the following section. In anticipation of that material some brief comments will be made now. In the autonomous (steady flow) case when $\partial \psi / \partial t=0$ particle trajectories coincide with streamlines, and the equations of motion (2) have analytic solutions involving integrals of functions of the streamfunction. However, in time-dependent flowseven simple time-periodic flows-chaotic motion may occur. Chaotic transport (Wiggins 1992; Rom-Kedar and Wiggins 1990; Rom-Kedar et al. 1990; Malhotra and Wiggins 1998; Coulliette and Wiggins 2001; Wiggins 2005; Miller et al. 1997, 2002) in flows described by Eqs. (2) is controlled by certain special material "lines" of fluid. Two types of such curves are particularly important: 1) stable and unstable manifolds of hyperbolic trajectories and 2) invariant tori predicted by the Kolmogorov-Arnold-Moser (KAM) theorem (Kolmogorov 1954; Arnold 1963; Rüssmann 1989; Jorba and Simó 1996; Sevryuk 2007). Both types of structure are composed of material lines of fluid and thus cannot be traversed by other fluid parcels, but the roles played by these structures in controlling transport are quite different. Loosely speaking, stable and unstable manifolds in nonsteady flows are involved in facilitating efficient transport, while KAM invariant tori are associated with transport barriers. For reasons that will be discussed below, numerically computed stable and unstable manifolds are usually referred to as Lagrangian coherent structures (LCSs) (Haller 2000; Haller and Yuan 2000; Haller 2001a,b; Haller 2002; Shadden et al. 2005). These ideas have been previously explored and applied to oceanographic transport problems (Coulliette and Wiggins 2001; Wiggins 2005; Lekien et al. 2005; Olascoaga et al. 2006; Poje and Haller 1999; Koshel and Prants 2006; Coulliette et al. 2007; Rogerson et al. 1999; Yuan et al. 2004; Samelson and Wiggins 2006; Samelson 1992; Mancho et al. 2008; Beron-Vera et al. 2008; Olascoaga et al. 2008). Transport in a double-gyre system has previously been studied in Coulliette and Wiggins (2001) and Poje and Haller (1999). In this paper we show that a new transport mechanism, which is not present in a two-gyre system, is necessary to explain transport in a three-gyre system.

The remainder of this paper is organized as follows. In section 2 we present an analytical model of a three-gyre system that is motivated by observations. Important theoretical results are then presented and illustrated in the context of this model. We provide an overview of relevant aspects of KAM theory, stable and unstable manifold structure, and lobe dynamics, emphasizing 
the complementary nature of these ideas as applied to the three-gyre system. Limitations of these theoretical results are also discussed. Also, we emphasize the qualitative change of behavior that occurs when the perturbation strength exceeds a critical value. Numerical results based on the idealized three-gyre system subject to a multiperiodic perturbation are presented to illustrate the importance of LCSs and the manner in which they control intergyre transport. In section 3 we turn our attention to a purely observation-based model of the Adriatic Sea, consisting of a surface-drifter-based estimate of the mean circulation on which a measured time-dependent altimetry-based perturbation is superimposed. It is demonstrated that all of the important qualitative LCS features described in section 2 can be identified in the observationally based model. Some shortcomings of our model are discussed. In section 4 we summarize and discuss our results. Some insights gained from the application of a dynamical-systemsbased approach to transport processes in a more general context are discussed.

\section{Transport in an idealized three-gyre model}

In this section we present theoretical material that relates to transport in a perturbed three-gyre system and present numerical simulations in an idealized threegyre system to illustrate several important concepts and results. We begin by presenting a steady streamfunction that is constructed from a time average of measured surface velocities in the Adriatic Sea. That observationally based streamfunction is then used to motivate our choice of an analytically described steady background three-gyre streamfunction. Then we use the analytically described background streamfunction with a time-dependent perturbation superimposed to perform numerical simulations. The analytically described model is well suited to this purpose, in part because dependence on parameters can be explored to illustrate important concepts and results. In the following section it will be shown that the important qualitative features emphasized in this section can be identified in a fairly realistic observationally based time-dependent model of the surface circulation of the Adriatic Sea.

Our first task in this section is to construct an observationally based estimate of the steady background streamfunction. Throughout this paper we assume that the flow is two-dimensional and incompressible so that Eqs. (2) are valid. Furthermore, we shall assume that the streamfunction can be expressed as the sum of a steady background and a time-dependent perturbation,

$$
\psi(x, y, t)=\psi_{0}(x, y)+\epsilon \psi_{1}(x, y, t) .
$$

The dimensionless perturbation strength $\epsilon$ need not be small. With the assumption that the temporal mean of the perturbation is zero, an observationally based estimate of $\psi_{0}(x, y)$ can be constructed from time averages of spatially binned measured velocities. This can be done by writing a finite difference approximation to the equations $\langle u\rangle=-\partial \psi_{0} / \partial y,\langle v\rangle=\partial \psi_{0} / \partial x$, followed by a least squares fitting procedure. Here $\langle u\rangle$ and $\langle v\rangle$ are the time-averaged measured $x$ and $y$ components of fluid velocity. The upper-left panel of Fig. 2 shows the result of such a calculation using surface-drifter-derived estimates of averaged velocities on a $0.1^{\circ}$ grid using overlapping $0.2^{\circ} \times 0.2^{\circ}$ bins. In that figure the geographical domain of the Adriatic has been rotated counterclockwise by about $45^{\circ}$, as was done in Fig. 1 . For convenience the origin of the coordinate system has been shifted. Note that $x$ increases from northwest to southeast. Also, the domain was closed by enforcing a no-flow condition through the Strait of Otranto (large $x$ ) and along smoothed coastlines. The very shallow shelf at the northern end of the Adriatic (small $x$ in Fig. 2) is excluded from consideration. Smoothing of the boundaries and the flow in close proximity to the boundaries does not influence the interior flow whose qualitative features, including the three-gyre structure, are consistent with the earlier discussion, based on Fig. 1. Additional caveats relating to $\psi_{0}(x, y)$ shown in the upper-left panel of Fig. 2, including limitations linked to the relevance of a temporal mean circulation, will be discussed in the next section.

The following simple analytically prescribed background streamfunction $\psi_{0}(x, y)$ is considered as an approximation to the surface-drifter-derived background streamfunction:

$$
\begin{aligned}
\psi_{0}(x, y)= & A \sin (C y)(E-\cos (B x)) \\
& \times\left\{\exp \left[D\left(x-L_{x}\right)\right]-1\right\}\left(X / L_{x}\right)^{F} .
\end{aligned}
$$

The values of the adjustable constants $L_{x}=600 \mathrm{~km}$, $L_{y}=150 \mathrm{~km}, A=2.62 \times 10^{3} \mathrm{~m}^{2} \mathrm{~s}^{-1}, B=6 \pi / L_{x}, C=$ $\pi / L_{y}, D=30 / L_{x}, E=3$, and $F=1 / 2$ were chosen to mimic the observationally based streamfunction. With these parameters streamlines corresponding to Eq. (4) are shown in the upper-right panel of Fig. 2. Note that $\psi=0$ at $x=0, x=L_{x}, y=0$, and $y=L_{y}$, so there is no flow through these boundaries. Despite its simplicity, this analytically prescribed background streamfunction reproduces the three-gyre structure of the Adriatic Sea and has correct length scales and time scales (periods of motion), as is evident from Fig. 2.

Stagnation points in a steady flow satisfy $u=v=0$. Stagnation points whose local topology is unchanged 

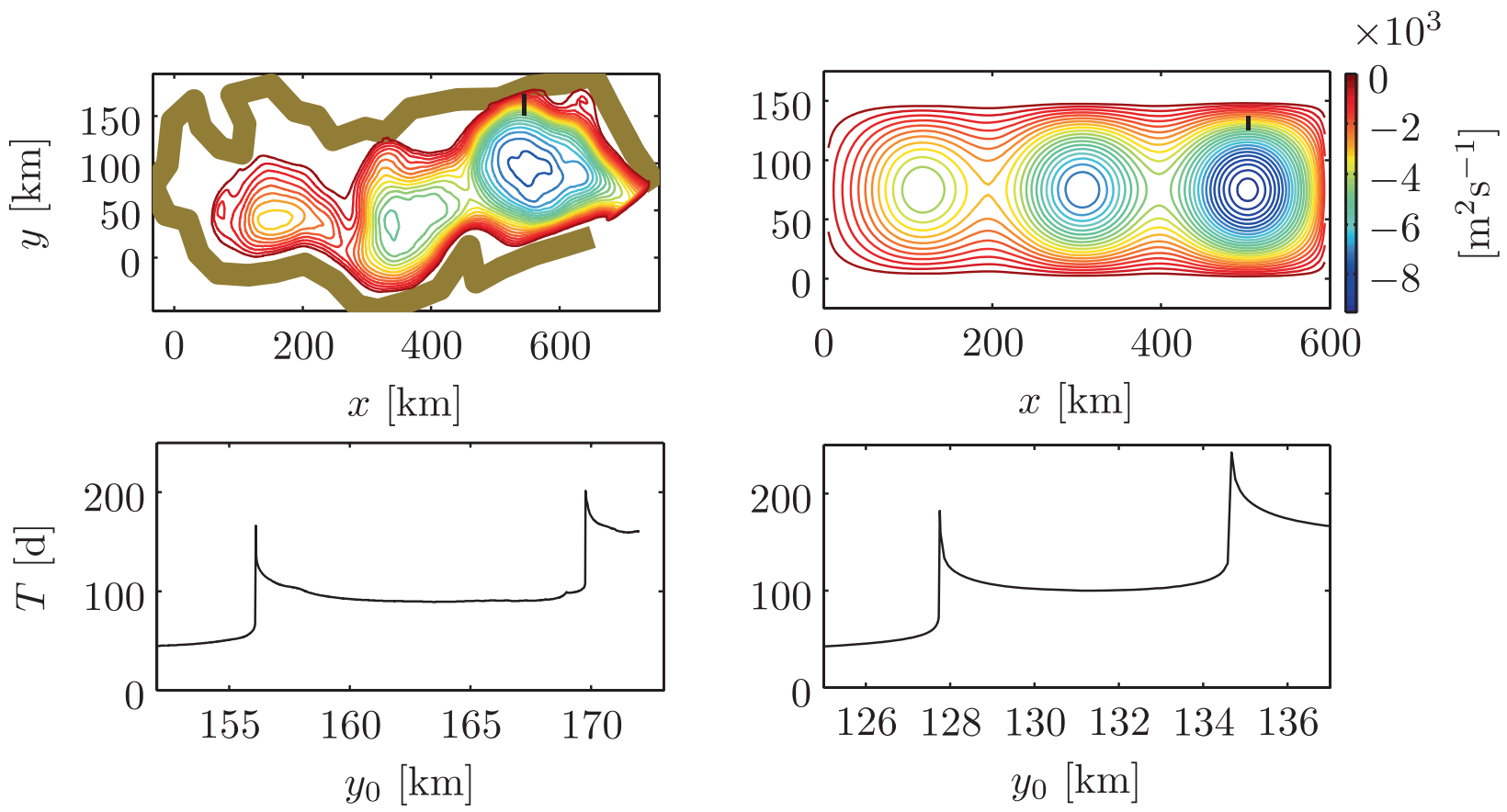

FIG. 2. (Upper left) Level surfaces of the streamfunction $\psi_{0}(x, y)$ that describe the mean surface circulation in the Adriatic Sea inferred from surface drifter trajectories. The thick brown line shows the smoothed boundary of the Adriatic Basin. Black dots at $x=545 \mathrm{~km}$ show the initial positions of the trajectories used to produce the lower-left plot. (Upper right) Level surfaces of the analytically specified streamfunction $\psi_{0}(x, y)$ given by Eq. (4). Black dots at $x=500 \mathrm{~km}$ show the initial positions of the trajectories used to produce the lowerright plot. (Lower) Periods of simulated trajectories $T$ for a family of trajectories with variable initial position $y_{0}$. (The period of a trajectory $T$ is the time taken for a trajectory to return to its initial position.) The initial positions of trajectories are marked in the plots above.

under a generic perturbation are of two types: 1) elliptic, corresponding to a local extremum of $\psi$, or 2) hyperbolic, corresponding to a local saddle in $\psi$. In the background three-gyre steady flow shown in Fig. 2, there are five stagnation points: three of the elliptic type that are located at the center of each gyre and two of the hyperbolic type that separate adjacent gyres from each other. Associated with each of the hyperbolic stagnation points are two trajectories, called homoclinic trajectories, that begin and end on the stagnation point. Each such homoclinic trajectory has an infinitely long period of motion. The homoclinic trajectories form two figure eights, one embedded inside the eastern loop of the other. The homoclinic trajectories separate regions of qualitatively different motion. The special case in which the two hyperbolic stagnation points fall on the same level surface of $\psi_{0}$ has a different topology consisting of two heteroclinic and two homoclinic trajectories. (In a steady flow, a heteroclinic trajectory begins and ends on different hyperbolic stagnation points.) That special case is not consistent with the observational database (Figs. 1 and 2) and will not be considered here.

Each of the lower panels of Fig. 2 shows periods of simulated trajectories, $T$, as a function of initial position for a set of trajectories that spans the two homoclinic trajectories. The lower-left panel was constructed using the observationally based streamfunction. The lower right panel was constructed using the analytically specified streamfunction. The initial positions of the simulated trajectories used in these calculations are shown in the upper panels of Fig. 2 with black dots (at $x=545$ and $x=500 \mathrm{~km}$, respectively). Three regions of qualitatively different motion can be identified in both panels of Fig. 2 based on the $T\left(y_{0}\right)$ structure shown: trajectories trapped inside the eastern gyre, trajectories going around the central and eastern gyre, and trajectories that go around all three gyres. Homoclinic trajectories emanating from two hyperbolic stagnation points separate these three regions of qualitatively different motion from one another. Note that $T \rightarrow \infty$ for those trajectories whose initial positions $y_{0}$ lie on the homoclinic trajectories. Between the two homoclinic trajectories $T\left(y_{0}\right)$ has a local minimum. We will return to this point below.

Note that the periods of simulated trajectories for our simple analytical streamfunction given by Eq. (4) are in good quantitative agreement with those produced by the streamfunction deduced from the drifter data. 
With the preceding observations as background, we now introduce some ideas that relate to the KolmogorovArnold-Moser theory, starting with the introduction of action-angle variables. For any steady streamfunction $\psi_{0}(x, y)$, the Lagrangian equations of motion (2) can be transformed to action-angle form [see, e.g., Landau and Lifshitz (1976) for details]. This transformation involves replacing the phase space coordinates $(x, y)$ with actionangle variables $(I, \theta)$ and replacing $\psi(x, y)$ with $H(I)$. In general, the transformation must be done piecewise in domains that are bounded by heteroclinic or homoclinic trajectories; there are five such domains in the steady flow shown in the upper-right panel of Fig. 2. After transforming to action-angle variables in each domain, the equations of motion are $d I / d t=-\partial H / \partial \theta=0, d \theta / d t=$ $\partial H / \partial I=\omega(I)$. By integrating these equations, one sees that $I$ is simply a label for a particular trajectory and that the motion is $2 \pi$ periodic in $\theta$ with angular frequency $\omega(I)$. A geometric picture (which also applies to higher dimensional Hamiltonian systems) is useful: phase space $(x, y)$ is foliated by a family of tori on which trajectories $(x(t), y(t))$ lie. The utility of this geometric picture stems, in part, from the fact that, as discussed below, some of these tori survive in the presence of certain types of timedependent perturbations.

The period of motion on a particular trajectory is connected to the angular frequency of motion in the usual fashion, $T(I)=2 \pi / \omega(I)$. The quantity $\omega^{\prime}(I)=d \omega /$ $d I$, which is a measure of shear on a particular trajectory in the background flow, will be important in our later discussion. The trajectory satisfying the condition $\omega^{\prime}(I)=$ 0 is referred to as shearless or twistless. For the analytical streamfunction, Eq. (4), plots of $T(I), \omega(I)$, and $\omega^{\prime}(I)$ for trajectories lying between two homoclinic trajectories are shown in Fig. 3. Note that in the vicinity of the homoclinic trajectories, $T(I)$ and $\omega^{\prime}(I)$ are large, while between the two homoclinic trajectories, $T(I)$ has a local minimum, $\omega(I)$ has a local maximum, and $\omega^{\prime}(I)=0$. In other words between the two figure eights formed by homoclinic trajectories lies a shearless trajectory, while the vicinity of the homoclinic trajectories is characterized by a large value of shear. These observations will be important in the discussion that follows.

We shall assume that the time-dependent perturbation $\psi_{1}(x, y, t)$ in Eq. (3) is a multiperiodic function of time, denoted symbolically as $\psi_{1}\left(x, y, \sigma_{1} t, \sigma_{2} t, \ldots, \sigma_{N} t\right)$. The number of frequencies present, $N$, is assumed to be finite and the frequencies are all nonzero. Without loss of generality, the frequencies can be assumed to be incommensurable (not rationally related), so $\psi_{1}$ is a quasiperiodic function of $t$. (The incommensurability assumption can be made because, if two or more frequencies are rationally related, the number of fre- quencies can be reduced. For example, a multiperiodic function with periods 4 and 6 weeks can be expressed as a simple periodic perturbation with period 12 weeks.) The assumption that $\psi_{1}$ is a multiperiodic function is made for two reasons. First, we note that in any enclosed basin any zero-mean time-dependent perturbation measured over a finite time interval-the perturbation streamfunction discussed in the following section, for example - can be accurately approximated as a multiperiodic function. This can be done, for example, by expressing $\psi_{1}(x, y, t)$ as a spatial empirical orthogonal expansion with time-dependent coefficients, $\psi_{1}(x, y, t)=\sum_{i} f_{i}(t) \Psi_{i}(x, y)$. The $\Psi_{i}(x, y)$ can be constructed in such a way that appropriate boundary conditions are satisfied. A harmonic decomposition of each $f_{i}(t)=\sum_{j} A_{i j} \cos \left(\sigma_{i j} t+\phi_{i j}\right)$ then yields a multiperiodic expansion of $\psi_{1}(x, y, t)$ with a finite number of terms that can be made to match measurements of $\psi_{1}(x, y, t)$ to whatever finite precision is desired. (Note that in the presence of noisy measurements it is generally not desirable to demand that misfit errors vanish.) Second, rigorous mathematical results-a KAM theorem (Kolmogorov 1954; Arnold 1963; Rüssmann 1989; Sevryuk 2007), in particular-apply to this class of problems. According to the KAM theorem for such systems (Jorba and Simó 1996; Sevryuk 2007), for a sufficiently weak perturbation and assuming certain other technical conditions are satisfied, some of the tori of the unperturbed system survive in the perturbed system. Those tori that survive under perturbation are referred to as KAM invariant tori. These surviving tori serve as impenetrable transport barriers (Rypina et al. 2007a). We will return to this point below.

The breakup of tori under perturbation is caused by the excitation and overlapping of resonances. Resonances are excited when the frequency of motion on the unperturbed torus $\omega(I)$ is rationally related to the vector frequency of the multiperiodic perturbation $\boldsymbol{\sigma}=\left\{\sigma_{1}\right.$, $\left.\ldots, \sigma_{N}\right\}$. The resonance condition is $n \omega\left(I_{0}\right)=\mathbf{m} \cdot \boldsymbol{\sigma}$, where $\mathbf{m}=\left\{m_{1}, \ldots, m_{N}\right\}$ with $n$ and $m_{i}, i=1,2, \ldots, N$ integers. Because generically a continuum of $\omega(I)$ is present, infinitely many resonances are exited even for a simple periodic perturbation, $N=1$. Among these many resonances the low-order resonances (such as 1:1 or 2:1) are the most important. Resonance widths are important because, when neighboring resonances overlap, the intervening tori break up; the widely used Chirikov definition of chaos is based on overlapping resonances (Chirikov 1979; Zaslavskii and Chirikov 1972; Lichtenberg and Lieberman 1983). Because resonances are excited at discrete values of $\omega$, it is the resonance width $\Delta \omega$ (rather than $\Delta I$ ) that controls whether neighboring resonances overlap. A simple analysis reveals (Chirikov 

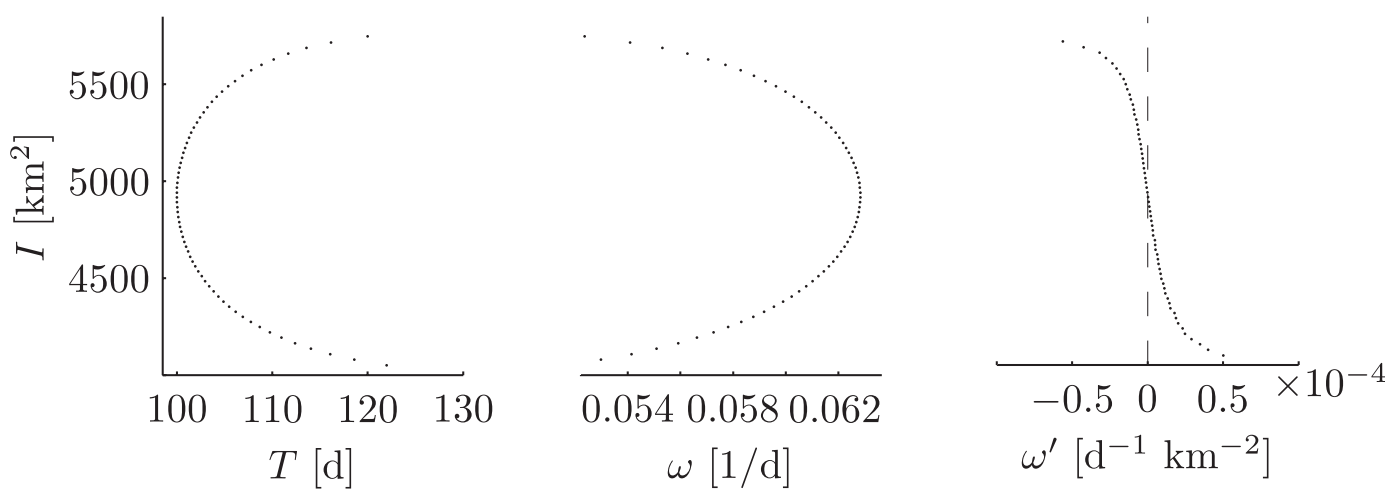

FIG. 3. Plots of (left) $T(I)$, (middle) $\omega(I)$, and (right) $\omega^{\prime}(I)$ for trajectories lying between the two homoclinic trajectories for the analytically described streamfunction, Eq. (4).

1979; Zaslavskii and Chirikov 1972; Rypina et al. 2007b) that resonance widths scale like

$$
\Delta \omega \sim \epsilon^{1 / 2}\left|\omega^{\prime}(I)\right|^{1 / 2},
$$

provided $\omega^{\prime}(I) \neq 0$. It follows from this expression that resonance widths $\Delta \omega$ are small near shearless tori where $\left|\omega^{\prime}(I)\right|$ vanishes. Recall from Fig. 3 that in our model of the Adriatic Sea a shearless torus is present between the figure eights discussed earlier. Because resonances that are excited near shearless tori have small resonance widths, these resonances are less likely to overlap than those for which $\left|\omega^{\prime}(I)\right|$ is larger. A consequence is that tori in the vicinity of shearless tori are expected to be resistant to breaking. The arguments just given lead to the notion of strong KAM stability near shearless (also known as degenerate) tori (Rypina et al. 2007a,b). On shearless tori a refined estimate of $\Delta \omega$ replaces Eq. (5) (Rypina et al. 2007b), but the qualitative behavior just described is unchanged by the refined estimate of $\Delta \omega$. It is important to emphasize, however, that the stability of tori near a shearless torus is not absolute. These tori will break up owing to a combination of two causes: 1) a loworder resonance may be excited in close proximity to the shearless torus, or 2) the strength of the perturbation $\epsilon$ may be too large.

Some of the results just described are illustrated with numerical simulations that are shown in the upper panels of Fig. 4. These numerical simulations use the analytically prescribed streamfunction, Eq. (4), and assume a particular form of a multiperiodic perturbation consisting of a superposition of standing waves:

$\psi_{1}(x, y, t)=\sum_{n, m} a_{n m} \cos \left(\sigma_{n m} t+\phi_{n m}\right) \sin \left(k_{x}^{n} x\right) \sin \left(k_{y}^{m} y\right)$ with $k_{x}^{n}=\pi n / L_{x}, k_{y}^{m}=\pi m / L_{y}$, so that the boundary condition $\psi=0$ at $x=0, x=L_{x}, y=0$, and $y=L_{y}$ is satisfied. The random phases $\phi_{n m}$ are uniformly distributed on $[0,2 \pi)$. The frequency spectrum is assumed to be red with $a_{n m} \sim \sigma_{n m}^{-1}$. The $a_{n m}$ were normalized so that, when $\epsilon=1$, the time-averaged spatially integrated kinetic energy associated with $\psi_{1}$ is equal to the spatially integrated kinetic energy associated with $\psi_{0}$. The wavenumber spectrum is assumed to be isotropic, $\sigma(\mathbf{k})=\sigma(|\mathbf{k}|)$. The latter assumption together with the geometrical constraint, $L_{x}=4 L_{y}$, leads to degenerate frequencies defined by the condition $\sigma_{n_{1}, m_{1}}=\sigma_{n_{2}, m_{2}}$. For $n_{\max }=8$ and $m_{\max }=2$, which are used for modeling purposes, two degenerate frequencies are present. Thus, for the perturbation considered, 14 different frequencies $\sigma_{n m}$ are present. For the numerical simulations shown here, the periods of standing waves in Eq. (6) were chosen to span the time interval from approximately 1 week to 2 months. Note that these periods are small compared to typical periods of rotation in the background flow (refer to Fig. 2).

The upper panels of Fig. 4 are Poincare sections. These plots reveal which of the original tori survive under perturbation and what structures are formed when tori are broken. For the class of flows considered here, the construction of a Poincare section requires that the perturbation $\psi_{1}$ be a simple periodic function of $t$ : the Poincare section is then constructed by plotting the positions of trajectories at integer multiples of the period of the perturbation. To satisfy the periodicity condition, the 14 standing wave periods were chosen to be commensurable with a common period of 60 days. This choice was made for the convenience of allowing Poincare sections to be constructed. It should be emphasized, however, that all of KAM theory results described in this paper (the theorem itself, resonance width estimates and arguments relating to strong KAM stability, and the absence of Arnold diffusion, as described below) hold for 

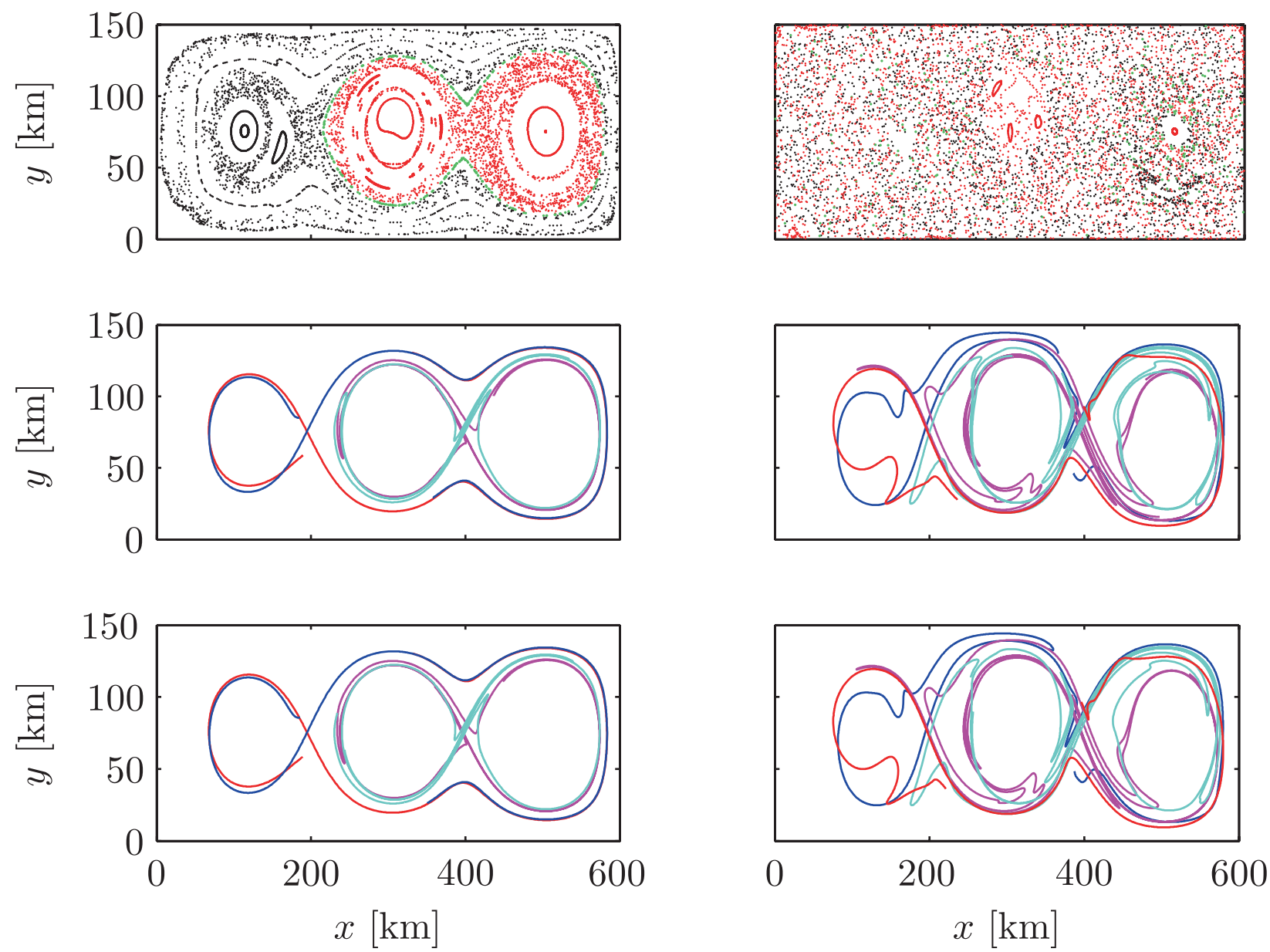

FIG. 4. Simulations based on the system described by Eqs. (2)-(4) and (6) for two values of $\epsilon$ : (left) $\epsilon=0.05$ and (right) $\epsilon=0.3$. (upper) Poincare sections for systems with a periodic perturbation comprising a superposition of 14 standing waves whose frequencies are commensurable with a common period of 60 days. (upper left) A KAM invariant torus is shown in green. Note that this closed curve serves as a transport barrier for the color-coded trajectories whose initial positions are inside (red dots) and outside (black dots) the closed curve. (middle) Stable (blue and light blue curves) and unstable (red and pink curves) manifolds of hyperbolic trajectories for the same systems that were used to produce the upper plots. (lower) Stable (blue and light blue curves) and unstable (red and pink curves) manifolds of hyperbolic trajectories for systems with a quasiperiodic perturbation comprising a superposition of 14 standing waves whose frequencies are not commensurable.

a general multiperiodic perturbation. We have chosen to show Poincare sections primarily because these figures nicely illustrate the phenomenon of strong KAM stability that we have described.

Consistent with the discussion above, Fig. 4 shows that for small $\epsilon$ some invariant tori (one such torus is shown in green in the upper-left panel) in close proximity to the shearless torus provide an impenetrable transport barrier that separates the western gyre from the central and eastern gyres. There appear to be two figure-eight-shaped chaotic bands, a large outer band and a smaller inner band inside the eastern loop of the outer band, that do not overlap. Consistent with our discussion of resonance widths and the "strong KAM stability" argument, the surviving tori (on which tra- jectories are nonchaotic) are observed in the vicinity of the shearless torus that lies between the two figure-eight bands. For large $\epsilon$ the barrier between these figure-eight bands is broken and a large chaotic region that includes portions of all three gyres is present. This is illustrated in the upper-right panel of Fig. 4. Again, we emphasize that, because the KAM theorem and the discussion above relating to resonances holds for a general multiperiodic perturbation, the relative robustness of the tori in the vicinity of the shearless torus holds for this larger class of perturbations.

In the Poincare sections shown in Fig. 4, the $x-y$ plane is partitioned into chaotic and nonchaotic (also known as "regular") regions. This partitioning is maintained even when the perturbation is sufficiently strong that all 
of the tori of the unperturbed system are broken. The regular regions form structures, often referred to as "islands," in an otherwise "chaotic sea." Island chains can be identified with resonances, for example, a dominant 5:1 resonance produces a five island chain. When many resonances are excited, a complicated island structure results. This partitioning of phase space into islands surrounded by a chaotic sea holds at arbitrarily small scales, that is, when small regions of the $x-y$ plane are blown up. For a general multiperiodic perturbation to a steady streamfunction, a Poincare section cannot be constructed, but for this class of problems $(x, y)$ at any $t$ is partitioned into chaotic and regular regions in the same way that a Poincare section is partitioned into chaotic and regular regions. This partitioning is a consequence of 1) the applicability of KAM theory to multiperiodically perturbed systems and, in particular, the observation that for this class of problems the excitation of resonances leads to both torus destruction and island formation; and 2) the absence of Arnold diffusion in such systems. Arnold diffusion is the process by which chaotic trajectories circumvent boundaries formed by collections of regular trajectories in autonomous Hamiltonian systems with three or more degrees of freedom (see, e.g., Wiggins 1992). The relevance of Arnold diffusion to our problem stems from the fact that a multiperiodically perturbed system of the type considered here with $N$ perturbation frequencies can be transformed to an autonomous system with $N+1$ degrees of freedom. The reason that Arnold diffusion does not occur in such systems is discussed in Rypina et al. (2007a) and Brown (1998). With the foregoing comments and the last sentence of the previous paragraph in mind, the Poincare sections shown in Fig. 4 should be seen as illustrative of qualitative properties common to the larger class of multiperiodic perturbations.

Now we turn our attention to a different, but complementary, set of concepts and tools from dynamical systems theory-stable and unstable manifolds and associated lobe structure - to provide additional insight into transport in our idealized three-gyre model. In nonsteady flows chaotic transport of fluids is controlled by stable and unstable manifolds of hyperbolic trajectories (Rom-Kedar and Wiggins 1990; Rom-Kedar et al. 1990; Wiggins 1992; Malhotra and Wiggins 1998; Coulliette and Wiggins 2001; Wiggins 2005). Stable and unstable manifolds are invariant curves (corresponding physically to material lines of fluid), so particle trajectories cannot cross these curves. Particle trajectories that start on the stable manifold approach the hyperbolic trajectory at an exponential rate as $t \rightarrow \infty$; particle trajectories that start on the unstable manifold ap- proach the hyperbolic trajectory at an exponential rate as $t \rightarrow-\infty$. In steady flows stable and unstable manifolds of hyperbolic points coincide with each other, forming homoclinic or heteroclinic trajectories. In nonsteady flows stable and unstable manifolds may intersect each other. Regions enclosed by segments of stable and unstable manifolds are called lobes. Lobes are important because fluid particles that are originally located inside the lobe are constrained to remain within the lobe as the flow evolves. When approaching one of the hyperbolic trajectories from which the stable or unstable manifold forming the lobe boundary emanates, the shape of the lobe becomes highly convoluted, while the enclosed area remains unchanged (in a 2D incompressible flow). The stable and unstable manifolds of hyperbolic trajectories, together with their intersections and associated lobes, provide a template for fluid exchange. These ideas will be illustrated below.

For flows with periodic time dependence (which need not be composed of a steady background plus a timedependent perturbation) hyperbolic trajectories and their stable and unstable manifolds can be unambiguously defined in terms of the eigenvalues of a linearized stability matrix (see, e.g., Wiggins 1992). For flows with general time dependence (which need not be multiperiodic or composed of a steady background plus a perturbation) hyperbolic trajectories and their stable and unstable manifolds can be defined by making use of a property known as "exponential dichotomy" (Yi 1993; Malhotra and Wiggins 1998). It is difficult to establish rigorously that this property is satisfied in flows with aperiodic time dependence. Owing to this difficulty, structures referred to as Lagrangian coherent structures, which can be computed numerically, have been defined (Haller 2000; Haller and Yuan 2000; Haller 2001a,b; Haller 2002; Shadden et al. 2005) in a way that is consistent with the exponential dichotomy property. Specifically, attracting (repelling) LCSs are defined (Shadden et al. 2005) as ridges of finite-time Lyapunov exponent (FTLE) fields computed in forward (backward) time. (The Lyapunov exponent is a measure of the rate at which neighboring trajectories diverge from one another.) Attracting and repelling LCSs serve as proxy stable and unstable manifolds, respectively. That FTLE-based estimates of LCSs serve as proxy stable and unstable manifolds can be tested numerically by computing the evolution of a dense set of trajectories that at some $t_{0}$ surround a hyperbolic trajectory; in forward (backward) time these trajectories follow the unstable (stable) manifold. Throughout this paper we use both FTLE calculations and the latter "direct manifold integration method" to numerically identify stable and unstable manifolds. 
We return now to a discussion of our idealized threegyre model of the Adriatic Sea, focusing on manifold structure. The qualitative change in the behavior of the perturbed three-gyre system associated with the breakup of the last surviving KAM torus that separates the central and eastern gyres from the western gyre when $\epsilon$ exceeds a certain threshold is accompanied by a qualitative change in the stable and unstable manifold structure. This is illustrated in the middle and lower panels of Fig. 4. In that figure segments of stable and unstable manifolds of hyperbolic trajectories are shown for the system $(2,3,4,6)$ for two values of $\epsilon$ : small $\epsilon(\epsilon=0.05)$ on the left and large $\epsilon(\epsilon=0.3)$ on the right. The segments of stable and unstable manifolds shown in the middle and lower panels of Fig. 4 were computed using the direct manifold integration method described above. The two middle panels of Fig. 4 correspond to a periodic perturbation $\psi_{1}$; the systems used to produce those plots are identical to those used to produce the Poincare sections shown in the upper panels. The two lower panels of Fig. 4 correspond to a quasiperiodic perturbation $\psi_{1}$ comprised of a superposition of 14 standing waves whose frequencies are not commensurable. Note that on both the left and right sides of Fig. 4 there is no qualitative difference in manifold structure between the periodically and quasiperiodically perturbed systems.

In each of the four lower subplots of Fig. 4 four stable/ unstable manifold pairs are evident: two stable/unstable manifold pairs are associated with each hyperbolic trajectory. In the middle and lower left panels of Fig. 4, which correspond to small $\epsilon$, stable and unstable manifolds associated with the same hyperbolic trajectory intersect each other. Manifold intersections of this type are called homoclinic intersections. The term homoclinic tangle is used to describe segments of manifolds that contain multiple homoclinic intersections. Homoclinic tangles are seen in the middle and lower left panels of Fig. 4. Each homoclinic tangle provides a template for chaotic mixing within the corresponding figure-eightshaped chaotic band. Intersections between stable and unstable manifolds associated with different hyperbolic trajectories are called heteroclinic intersections. No heteroclinic intersections are seen in the middle and lower left panels of Fig. 4, corresponding to small $\epsilon$. This is consistent with the upper left panel of Fig. 4, which shows two chaotic figure-eight bands separated by an impenetrable barrier. That barrier prevents heteroclinic manifold intersections from occurring. In the middle and lower right panels of Fig. 4, $\epsilon$ is sufficiently large that this barrier is broken; both homoclinic and heteroclinic intersections between stable and unstable manifolds are present. Heteroclinic tangles and associated heteroclinic lobes provide a template for fluid exchange between all three gyres. This is consistent with the upper right panel of Fig. 4 where one well-mixed chaotic region includes portions of all three gyres.

Owing to time periodicity of the perturbation, the manifold structure in the middle panels of Fig. 4 repeats itself after each period of the perturbation $2 \pi / \sigma$. This is not true for the two lower panels of Fig. 4, where the manifold structure evolves in an aperiodic fashion. Despite this difference, the qualitative similarity between the middle and lower panels of Fig. 4 strongly suggests that qualitative features of the manifold structure in the perturbed system are largely controlled by the background streamfunction, rather than details of the perturbation.

In this section we have discussed the theory and application of dynamical system tools to the study of transport in an idealized, analytically prescribed, threegyre system. A qualitative change in transport properties of the perturbed three-gyre system was shown to occur when the perturbation strength exceeds a certain threshold. This change is associated with the breakup of the last surviving KAM invariant torus in the vicinity of the shearless torus and the corresponding appearance of heteroclinic manifold intersections. For $\epsilon$ less than the critical value, KAM invariant tori near the shearless torus serve as a transport barrier between two figureeight-shaped bands associated with the chaotic motion driven by stable and unstable manifolds of the two hyperbolic trajectories; under such conditions, all manifold intersections are of the homoclinic type. When $\epsilon$ exceeds the critical value, there are no remaining KAM invariant tori in the vicinity of the unperturbed shearless torus and a large chaotic region is formed that includes both hyperbolic trajectories and portions of all three gyres: Under such conditions, heteroclinic manifold intersections are formed and the associated lobe structure provides a template for transport within portions of all three gyres. These qualitative features are illustrated schematically in Fig. 5. The critical value of $\epsilon$ at which the bifurcation occurs depends on details of both the background structure $\psi_{0}(x, y)$ and the perturbation $\psi_{1}(x, y, t)$. Because of this, numerical simulations are, in general, required to estimate the critical perturbation strength. Despite this, knowledge that the qualitative features described here are robust (discussed in more detail in the following section) and that the critical value of $\epsilon$ is finite are both important.

\section{Transport in an observationally based three-gyre model}

In this section an observationally based model of the surface circulation of the Adriatic Sea is used to test, in 


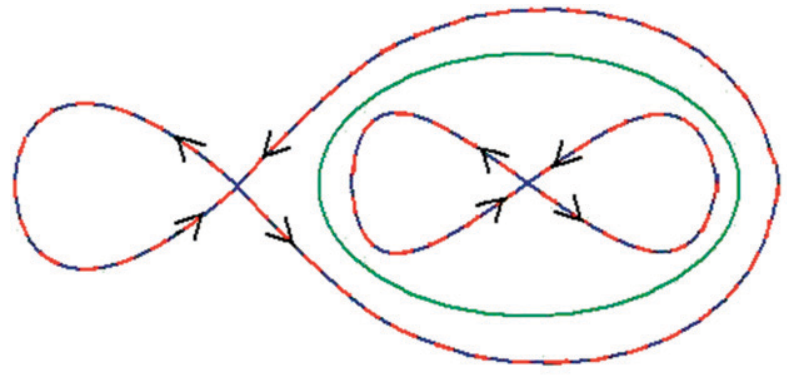

$\psi_{0}(x, y)$ and $\psi_{1}(x, y, t)$ allows dependence on the perturbation strength $\epsilon$ in Eq. (3) to be explored. In our simulations $\epsilon=1$ corresponds to the true perturbation strength. The measured altimetry-based perturbation field covers the time interval of one year (from $t=0$ on 6 January 1993 to $t=365$ days on 5 January 1994) with $\Delta t=7$ days. Measured height fields were modified in a thin boundary layer around the perimeter of the domain to enforce the condition $\psi_{1}=0$ on the smoothed coastline. For the true perturbation strength $\epsilon=1$, the ratio of the time-averaged integrated kinetic energy associated with $\psi_{1}$ to the integrated kinetic energy associated with $\psi_{0}$ is 1.05 . Thus, to a good approximation, $\epsilon$ can be thought of as the ratio of transient (or "eddy") kinetic energy to mean kinetic energy.

Before proceeding, it should be noted that, despite both $\psi_{0}(x, y)$ and $\psi_{1}(x, y, t)$ being observationally based, their sum has some rather obvious shortcomings as a model of the surface circulation of the Adriatic. First, the surface circulation in the Adriatic Sea is strongly influenced by intense short-lived bora and sirocco wind forcing events (Ursella et al. 2007). During these events the decomposition of Eq. (3) might be questioned. Second, the measured velocity field associated with the altimetry-based perturbation need not coincide with near-surface currents. Third, the gridded altimetrybased perturbation height field $h(x, y, t)$ on which our calculations are based is sampled at approximately $6 \mathrm{~km}$ spatially and 1 week temporally. Variability with shorter length and time scales (including submesoscale and possibly also some short mesoscale structure) is not resolved. Fourth, tide removal from altimetry-based observations is difficult in enclosed basins such as the Adriatic, leading unavoidably to some aliasing of tidal energy into mesoscale fields. Fifth, our smoothing of the coastlines and neglect of transport through the Strait of Otranto are rather severe approximations. For these reasons the velocity field described by the sum, $\psi_{0}(x, y)+$ $\psi_{1}(x, y, t)$, should not be thought of as an accurate deterministic description of the surface circulation of the Adriatic Sea within the relevant time window. Rather, this velocity field should be thought of as describing typical, mostly mesoscale, near-surface current variability throughout the interior of the Adriatic Sea.

The altimetry-based perturbation field $\psi_{1}(x, y, t)$ is

a more realistic setting, the robustness of the qualitative features that were emphasized in the previous section. The streamfunction used has the form of Eq. (3), where $\psi_{0}(x, y)$ is the surface-drifter-derived mean streamfunction shown in Fig. 2 and $\psi_{1}(x, y, t)=(g / f) h(x, y, t)$, where $h(x, y, t)$ is a measured altimetry-based perturbation to sea level height, $g$ is gravitational acceleration, and $f$ is the local Coriolis parameter. Knowledge of both not a periodic function of $t$, so it is not possible to construct a Poincare section for the flow described by $\psi_{0}(x, y)+\epsilon \psi_{1}(x, y, t)$. However, $\psi_{1}$ can be expanded as a multiperiodic function of time (using the method described in the previous section, for example), so the KAM theory-based arguments presented in the previous section are still applicable. With this in mind, we expect that all of the important qualitative features of 
the perturbed three-gyre system that were discussed in the preceding section should hold for the observationally based system considered here. In particular, we seek to test whether the following qualitative features of the manifold and lobe structure, which were emphasized in the previous section, can be reproduced using the measured velocity fields: 1) for small $\epsilon$ all manifold intersections are of the homoclinic type and manifolds form two nonoverlapping figure-eight-shaped bands, each with a crossing region that covers one of the hyperbolic trajectories; and 2) for large $\epsilon$ the transport barrier between the two figure-eight bands is broken and heteroclinic manifold intersections are formed, thereby allowing transport between all three gyres.

Before proceeding, we wish to emphasize that our concern in this section is with the manifold structure associated with the basin-scale circulation in the Adriatic. This structure is linked to the two dominant hyperbolic trajectories in proximity to the two hyperbolic stagnation points in the background steady flow (recall the upper-left panel of Fig. 2). (Note, however, that the hyperbolic trajectories from which stable and unstable manifolds emanate need not coincide with hyperbolic stagnation points that appear in snapshots of the streamfunction, as the locations of stagnation points are sensitive to the choice of reference frame.) We exclude from consideration in this section localized structures associated with hyperbolic trajectories near the northwestern end of the smoothed Adriatic domain and the localized structures near the centers of the three main gyres. The structures that we neglect are important locally, but they do not influence the larger-scale intergyre transport that is the focus of our study.

Numerical simulations based on the observationally based model described above are shown in Figs. 6-8. For the two values of $\epsilon$-small $\epsilon(=0.1)$ on the left and large $\epsilon(=1)$ on the right-stable and unstable manifolds of hyperbolic trajectories are shown inFig. 6 . The upper four subplots in this figure show finite time Lyapunov exponent (FTLE) estimates computed in forward (upper two subplots) and backward (middle two subplots) time as a function of initial condition. (Recall that FTLEs are a measure of the rate at which neighboring passively advected fluid particles separate from each other over a finite time interval.) Regions of most intense red in the upper two and middle two subplots of Fig. 6 correspond to stable and unstable manifolds of hyperbolic trajectories. The lower two subplots of Fig. 6 show segments of stable and unstable manifolds constructed using the direct manifold integration method described above. Unstable manifolds of the two hyperbolic points are shown using red and pink curves; stable manifolds are shown using blue and light blue curves. Note that manifold estimates computed as maximizing ridges of the FTLE field are in good agreement with manifolds computed using the direct manifold integration method.

Consistent with the behavior of the previously described idealized three-gyre system under perturbation and in qualitative agreement with Fig. 4, both methods serve to confirm that in the small $\epsilon$ case (left three panels of Fig. 6) all manifold intersections are of the homoclinic type. This strongly suggests the existence of two nonoverlapping figure-eight bands of chaotic motion, each with a crossing region that covers one of the hyperbolic trajectories. Chaotic motion inside each figure-eight band is governed by lobes produced by homoclinic intersections of stable and unstable manifolds of the corresponding hyperbolic trajectory. The absence of heteroclinic intersections indicates that the two figureeight bands do not exchange fluid with each other and suggests the existence of a barrier to transport between the two figure-eight bands. In the large $\epsilon$ case, illustrated in the three right panels of Fig. 6, both the FTLE field and the direct manifold integration calculation indicate the presence of manifold intersections of the heteroclinic type. Associated with these intersections and the associated lobes is one large chaotic region that includes portions of all three gyres. Lobes formed by heteroclinic intersections of manifolds provide a template for gyre-to-gyre-to-gyre transport within this large chaotic region.

A lobe formed by heteroclinic intersections of stable and unstable manifolds is readily identifiable in the lower-right plot in Fig. 6. The evolution of this lobe in forward and backward time is shown in Fig. 7. In forward time that portion of the boundary of the lobe that comprises a segment of the stable manifold dramatically shrinks as the manifold is pulled toward the western hyperbolic trajectory. Similarly, in backward time that portion of the boundary of the lobe that comprises a segment of the unstable manifold dramatically shrinks as the manifold is pulled toward the eastern hyperbolic trajectory. As these segments of the boundary of the lobe contract or expand, the area enclosed within the lobe remains constant. (This is a consequence of the assumption of $2 \mathrm{D}$ incompressible flow.) Lobes formed by homoclinic intersections of stable and unstable manifolds are also present in the flow; portions of the boundary of these lobes approach the same hyperbolic trajectory in forward and backward time.

Intergyre transport is illustrated in Fig. 8, which shows how initially compact distributions of passive tracers evolve in time for both small and large $\epsilon$. For small $\epsilon$ tracer particles do not cross the transport barrier, and the central and eastern gyres are isolated from the 

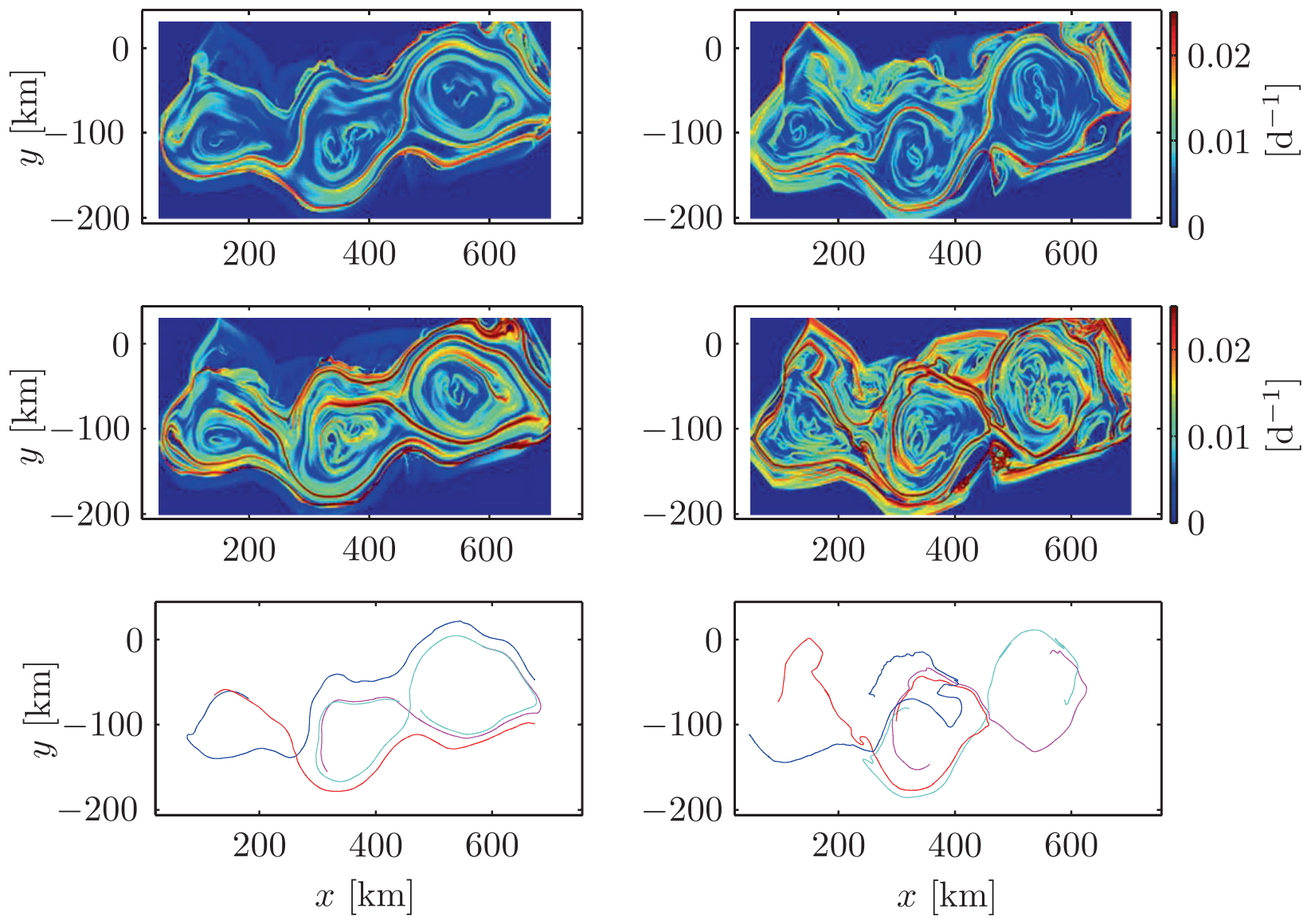

FIG. 6. Simulations of stable and unstable manifolds using the observationally based model of the Adriatic Sea for two values of the perturbation strength: (left) $\epsilon=0.1$ and (right) $\epsilon=1$. (upper) FTLE estimates computed in forward time relative to $t=182$ days. Ridges of intense red correspond to stable manifolds. (middle) FTLE estimates computed in backward time relative to $t=182$ days. Ridges of intense red correspond to unstable manifolds. (lower) Stable (blue and light blue curves) and unstable (red and pink curves) manifolds computed using the direct manifold integration method relative to $t=182$ days. Note that stable and unstable manifolds computed using FTLEs and the direct manifold integration method are in excellent agreement.

western gyre. For large $\epsilon$, however, the transport barrier is broken, and there is a significant exchange of fluid between the central and eastern gyres and the western gyre.

Note that, because of the complicated time dependence of $\psi_{1}(x, y, t)$, the results shown in Figs. 6-8 depend on the starting time chosen. Numerical simulations have shown that the qualitative behavior that we have emphasized throughout this section is not sensitive to the start time chosen.

Consistent with the results presented in the previous section, we have seen in this section that lobes formed by intersecting stable and unstable manifolds are present in the flows shown on both left (small $\epsilon$ ) and right (large $\epsilon$ ) sides of Fig. 6. Associated with these lobes is chaotic transport, subject to the caveat noted in the final section. There is a fundamental difference, however, between the small and large $\epsilon$ flows. For small $\epsilon$ the transport barrier surrounding the central and eastern gyres isolates these gyres from the western gyre. Under these conditions all manifold intersections are of the homoclinic type. For large $\epsilon$ the transport barrier is broken and heteroclinic manifold intersections are formed. It is the presence of these heteroclinic manifold intersections and the associated lobes that facilitates transport between the central and eastern gyre, and the western gyre.

\section{Summary and discussion}

Motivated by observations of surface drifter trajectories in the Adriatic Sea, we have studied fluid transport in a steady three-gyre system subject to a timedependent perturbation. Our study has relied heavily on dynamical systems tools and concepts: KAM theory, stable and unstable manifolds and lobe structure, and 

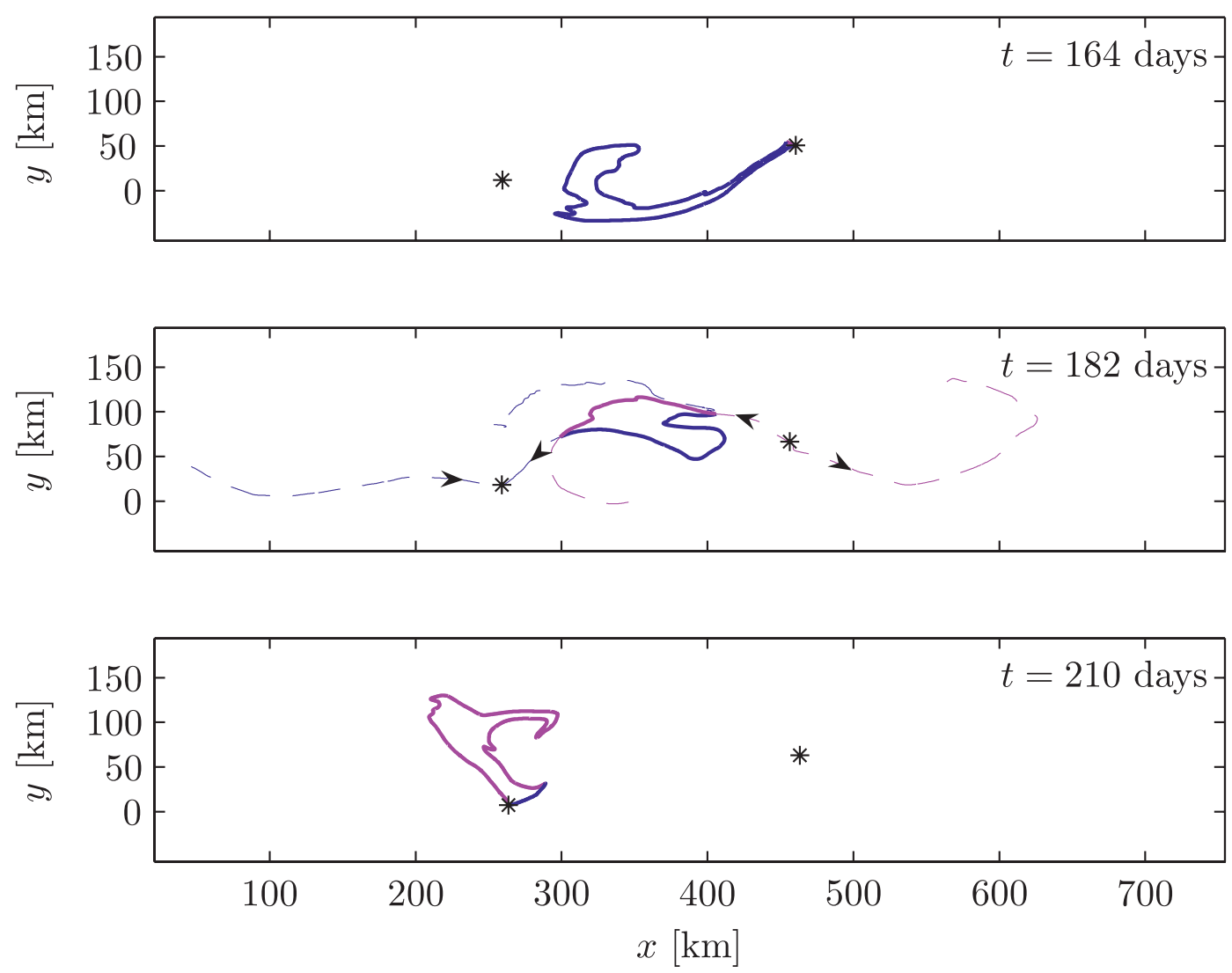

FIG. 7. Simulations of the evolution of a heteroclinic lobe in forward and backward time relative to $t=182$ days using the observationally based model of the Adriatic Sea with the true value of the perturbation strength, $\epsilon=1$. The boundary of the heteroclinic lobe is shown at the times indicated. The portion of the boundary of the lobe that comprises a segment of the unstable (stable) manifold is shown in pink (blue). Positions of the two (eastern and western) hyperbolic trajectories are shown with asterisks. (middle) The unstable and stable manifolds, which form the heteroclinic lobe, are shown by dashed pink and blue lines, respectively. Arrows on the manifolds indicate the direction of attraction/repulsion.

evolution. An analytically specified model was introduced to illustrate the importance of KAM invariant tori, stable and unstable manifolds, and the manner by which these structures control transport. A fully observationally based model of the Adriatic Sea surface circulation was used to demonstrate the robustness of the important qualitative features that were observed in the analytical model.

It was shown using both the analytic model and the observationally based model that the perturbed threegyre system has qualitatively different behavior for small and large perturbation. For small perturbation 1) a transport barrier consisting of KAM invariant tori isolates the central and eastern gyres from the western gyre, 2) all stable and unstable manifold intersections are of the homoclinic type, and 3) there is no gyre-togyre-to-gyre transport. For large perturbation 1) the transport barrier, composed of a thin band of KAM invariant tori, that separates the central and eastern gyres from the western gyre is broken; 2) both homoclinic and heteroclinic manifold intersections are present; and 3) it is the lobe structure associated with heteroclinic manifold intersections that facilitates gyreto-gyre-to-gyre transport. We note also that there is a close connection and consistency between the KAM theory arguments that we have presented and the arguments based on manifolds and lobes.

The simulations that we have performed in the observationally based model of the Adriatic Sea indicate that the true perturbation strength $(\epsilon=1)$ corresponds to the strongly perturbed regime in which transport between all three gyres occurs. This statement is consistent (superficially, at least) with Fig. 1, which strongly suggests that transport between all three gyres occurs. It is not surprising that the surface circulation of the Adriatic Sea corresponds to the strongly perturbed regime in our models. The utility of our study lies in the insight provided into the underlying dynamical 

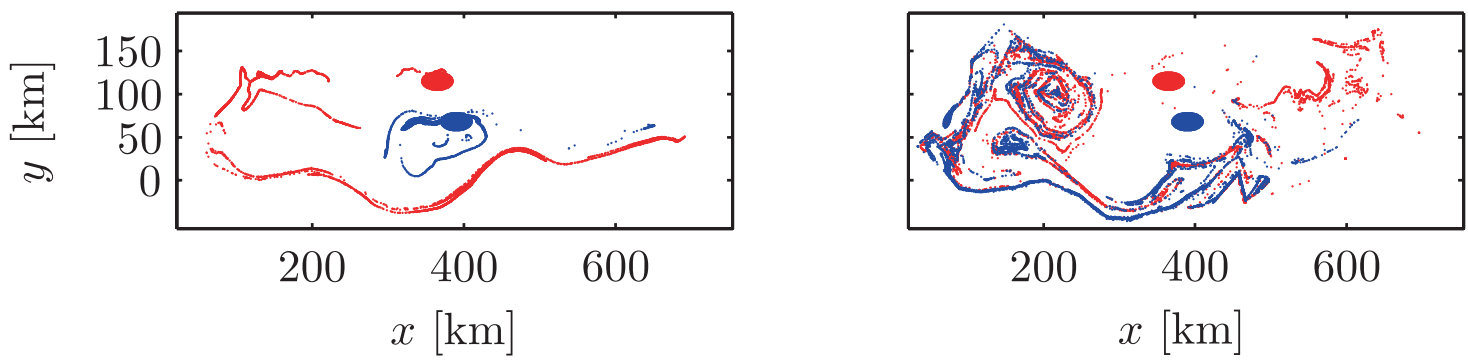

FIG. 8. Initial $(t=182)$ and final $(t=302$ days $)$ position of two sets of passive tracers in the observationally based model of the Adriatic Sea for two values of the perturbation strength: (left) $\epsilon=0.1$ and (right) $\epsilon=1$. The two sets of tracers are color coded: red and blue. The initial positions of the two sets of tracers lie inside two circles. Note that for $\epsilon=0.1$ there is no mixing (in a coarse-grained sense) of red and blue tracers, while for $\epsilon=1$ there is strong mixing.

processes that allow gyre-to-gyre-to-gyre transport to take place in the Adriatic Sea or any other perturbed three-gyre system. We have shown that critical elements of these dynamics are the breaking of a transport barrier and the formation of heteroclinic manifold intersections when the perturbation strength exceeds a certain threshold. These dynamical elements play no role in a perturbed double-gyre system (Coulliette and Wiggins 2001; Poje and Haller 1999).

There is a growing appreciation that dynamical system tools and concepts are extremely useful and insightful in studies involving fluid transport when the velocity field (in our case, the streamfunction) is known. Under such conditions the space-time structure of stable and unstable manifolds and the associated lobe structure can be computed (recall Figs. 6 and 7), and transport can be quantified. What is less appreciated is that, even when only qualitative information about the velocity field is known, the same dynamical system tools can provide critically important qualitative information about transport. The qualitative properties described in the previous paragraphs illustrate this point.

Our interpretation of the numerical results that we have presented rests on the relevance of two sets of mathematical results to the oceanographic problem that we have investigated. These mathematical results relate to the Kolmogorov-Arnold-Moser (KAM) theory and the stable and unstable manifolds of hyperbolic trajectories. As noted previously, both sets of results have shortcomings. KAM theory has two principal shortcomings: 1) it is a perturbation theory based on the assumption that the decomposition (3) is valid with $\epsilon$ small, and 2) the least restrictive form of the theorem proved to date (Sevryuk 2007) assumes that the perturbation streamfunction has quasiperiodic time dependence. (No KAM theorem has been proved for general aperiodic time dependence.) Because our focus was on understanding the qualitative difference in behavior between small and large $\epsilon$, the small $\epsilon$ restriction was not, for our purposes, critical. (Note also that numerical simulations in many systems reveal that some invariant tori that are predicted by the theorem persist for $\epsilon \simeq 1$, so the small $\epsilon$ is less restrictive than one might expect.) The restriction to quasiperiodic time dependence necessitated that we argue that the perturbation be expressible as a multiperiodic function of time. In contrast to KAM theory, results relating to stable and unstable manifolds of hyperbolic trajectories are valid for flows with general aperiodic time dependence. For this class of flows, the exponential dichotomy property provides a rigorous basis for defining hyperbolic trajectories and their stable and unstable manifolds. Unfortunately, apart from this defining property, few rigorous results have been established for general aperiodic flows. [Note, e.g., that the term "chaos" is used in a very restricted fashion by mathematicians and that only very restricted classes of systems have been shown rigorously to possess chaotic dynamics; see, e.g., section 1.2.2 of Mancho et al. (2006) and references therein; Wiggins (1999); and Palmer and Stoffer (1989).] It is precisely this void that has provided the stimulation for recent work relating to LCSs (Haller 2000; Haller and Yuan 2000; Haller 2001a,b; Haller 2002; Shadden et al. 2005). This type of analysis has proven to be extremely useful in applications (Lekien et al. 2005; Olascoaga et al. 2006; Mathur et al. 2007; Koshel and Prants 2006; Coulliette et al. 2007; Yuan et al. 2004; Rogerson et al. 1999; Beron-Vera et al. 2008; Olascoaga et al. 2008) but it relies heavily on numerical calculations and has its own limitations (Shadden et al. 2005). In this study we have shown that, despite some shortcomings associated with both KAM theory and LCSs, both sets of ideas provide critically important insight into the problem we have studied-transport in a three-gyre system.

The qualitative features of the three-gyre problem that we have described are expected to hold for a large class of multigyre steady flows $\psi_{0}(x, y)$ subject to a time- 
dependent perturbation $\epsilon \psi_{1}(x, y, t)$. For small $\epsilon$ transport barriers of the strong KAM stability type are expected to be present and isolate some gyres from the remainder of the flow. As $\epsilon$ is increased, barriers of this type will break and heteroclinic intersections of manifolds will form, thereby facilitating intergyre transport. These heteroclinic intersections are critically important in facilitating efficient long-range transport of fluid. The more strongly the background flow is perturbed, the more prevalent heteroclinic intersections of manifolds will become as transport barriers break. This suggests that, quite generally, one might identify turbulent transport (Mathur et al. 2007) as being characterized by an abundance of heteroclinic manifold intersections.

Acknowledgments. We thank F. J. Beron-Vera, M. J. Olascoaga, and K. Palmer for the benefit of useful discussions relating to dynamical systems and fluid transport. We thank P.-M. Poulain for providing surface drifter-derived average surface currents in the Adriatic Sea that were used in this study. The altimetric products used in this study were produced by SSALTO/DUACS and distributed by AVISO, with support from CNES. We thank M. J. Olascoaga and J. Trinanes for providing assistance in obtaining these data. This work was supported by the National Science Foundation Grants CMG0417425 and CMG0825547.

\section{REFERENCES}

Arnold, V. I., 1963: Proof of a theorem of A. N. Kolmogorov on the invariance of quasi-periodic motions under small perturbations of the Hamiltonian. Russ. Math. Surv., 18, 9-36.

Artegiani, A., D. Bregant, E. Pashini, N. Pinardi, F. Raicich, and A. Russo, 1997a: The Adriatic Sea general circulation. Part I: Air-sea interactions and water mass structure. J. Phys. Oceanogr., 27, 1492-1514.

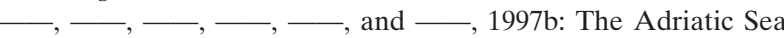
general circulation. Part II: Baroclinic circulation structure. $J$. Phys. Oceanogr., 27, 1515-1532.

Beron-Vera, F. J., M. J. Olasocaga, and G. J. Goni, 2008: Oceanic mesoscale eddies as revealed by Lagrangian coherent structures. Geophys. Res. Lett., 35, L12603, doi:10.1029/ 2008 GL033957.

Brown, M. G., 1998: Phase space structure and fractal trajectories in $1 \frac{1}{2}$ degree of freedom Hamiltonian systems whose time dependence is quasiperiodic. Nonlinear Processes Geophys., $\mathbf{5}, 69-74$.

Castellari, S., A. Griffa, T. M. Ozkomen, and P.-M. Poulain, 2001: Prediction of particle trajectories in the Adriatic Sea using Lagrangian data assimilation. J. Mar. Syst., 29, 33-50.

Chirikov, B. V., 1979: A universal instability of many-dimensional oscillator systems. Phys. Rep., 52, 265-379.

Coulliette, C., and S. Wiggins, 2001: Intergyre transport in a winddriven, quasigeostrophic double gyre: An application of lobe dynamics. Nonlinear Processes Geophys., 8, 69-94.

— , F. Lekien, J. D. Paduan, G. Haller, and J. E. Marsden, 2007: Optimal pollution mitigation in Monterey Bay based on coastal radar data and nonlinear dynamics. Environ. Sci. Technol., 41, 6562-6572.

Cushman-Roisin, B., M. Gačić, P.-M. Poulain, and A. Artegiani, 2001: Physical Oceanography of the Adriatic Sea: Past, Present and Future. Kluwer Academic, 304 pp.

Falco, P., A. Griffa, P.-M. Poulain, and E. Zambianchi, 2000: Transport properties of the Adriatic Sea as deduced from drifter data. J. Phys. Oceanogr., 30, 2055-2071.

Haller, G., 2000: Finding finite-time invariant manifolds in twodimensional velocity fields. Chaos, 10, 99-108.

, 2001a: Distinguished material surfaces and coherent structures in 3D fluid flows. Physica D, 149, 248-277.

2001b: Lagrangian structures and the rate of strain in a partition of two-dimensional turbulence. Phys. Fluids, 13, $3365-3385$.

, 2002: Lagrangian coherent structures from approximate velocity data. Phys. Fluids, 14, 1851-1861.

— , and G. Yuan, 2000: Lagrangian coherent structures and mixing in two-dimensional turbulence. Physica D, 147, 352-370.

Haza, A. C., L. I. Piterbarg, P. Martin, T. M. Ozgokmen, and A. Griffa, 2007a: A Lagrangian subgridscale model for particle transport improvement and application in the Adriatic Sea using the Navy Ocean Model. Ocean Modell., 17, 68-91.

_ , and Coauthors, 2007b: Model-based directed drifter launches in the Adriatic Sea: Results from DART experiment. Geophys. Res. Lett., 34, L10605, doi:10.1029/ 2007 GL029634.

Jorba, Á., and C. Simó, 1996: On quasi-periodic perturbations of elliptic equilibrium points. SIAM J. Math. Anal., 27, 17041737.

Kolmogorov, A. N., 1954: On the persistence of conditionally periodic motions under a small change of the Hamilton function (in Russian). Dokl. Akad. Nauk SSSR, 98, 525-530.

Koshel, K. V., and S. V. Prants, 2006: Chaotic advection in the ocean. Phys.-Usp., 49, 1151-1178.

Lacorata, G., E. Aurell, and A. Vulpiani, 2001: Drifter dispersion in the Adriatic Sea: Lagrangian data and chaotic model. Ann. Geophys., 19, 121-129.

Landau, L. D., and E. M. Lifshitz, 1976: Mechanics. 3rd ed. Course of Theoretical Physics, Vol. 1, Pergamon, 169 pp.

Lekien, F., C. Coulliette, A. J. Mariano, E. H. Ryan, L. K. Shay, G. Haller, and J. E. Marsden, 2005: Pollution release tied to invariant manifolds: A case study for the coast of Florida. Physica D, 210, 1-20.

Lichtenberg, A. G., and M. A. Lieberman, 1983: Regular and Stochastic Motion. Springer-Verlag, 499 pp.

Malhotra, N., and S. Wiggins, 1998: Geometric structures, lobe dynamics, and Lagrangian transport in flows with aperiodic time-dependence, with applications to Rossby wave flow. $J$. Nonlinear Sci., 8, 401-456.

Mancho, A. M., D. Small, and S. Wiggins, 2006: A tutorial on dynamical systems concepts applied to Lagrangian transport in oceanic flows defined as finite time data sets: Theoretical and computational issues. Phys. Rep., 437, 55-124.

, E. Hernández-García, D. Small, S. Wiggins, and V. Fernández, 2008: Lagrangian transport through an ocean front in the northwestern Mediterranean Sea. J. Phys. Oceanogr., 38, 1222-1237.

Mathur, M., G. Haller, T. Peacock, J. E. Ruppert-Felsot, and H. L. Swinney, 2007: Uncovering the Lagrangian skeleton of turbulence. Phys. Rev. Lett., 98, Abstract 144502.

Maurizi, A., A. Griffa, P.-M. Poulain, and F. Tampieri, 2004: Lagrangian turbulence in the Adriatic Sea as computed from 
drifter data: Effects of inhomogeneity and nonstationarity. $J$. Geophys. Res., 109, C04010, doi:10.1029/2003JC002119.

Miller, P. D., C. K. R. T. Jones, A. M. Rogerson, and L. J. Pratt, 1997: Quantifying transport in numerically generated velocity fields. Physica D, 110, 105-122.

- L. J. Pratt, K. R. Helrich, and C. K. R. T. Jones, 2002: Chaotic transport of mass and potential vorticity for an island recirculation. J. Phys. Oceanogr., 32, 80-102.

Olascoaga, M. J., I. I. Rypina, M. G. Brown, F. J. Beron-Vera, H. Koçak, L. E. Brand, G. R. Halliwell, and L. K. Shay, 2006: Persistent transport barrier on the West Florida Shelf. Geophys. Res. Lett., 33, L22603, doi:10.1029/2006GL027800.

, F. J. Beron-Vera, L. E. Brand, and H. Kocak, 2008: Tracking the early development of harmful algal blooms on the West Florida Shelf with the aid of Lagrangian coherent structures. J. Geophys. Res., 113, C12014, doi:10.1029/2007JC004533.

Orlic, M., M. Gacic, and P. E. La Violette, 1992: The currents and circulation of the Adriatic Sea. Oceanol. Acta, 15, 109-124.

Palmer, K., and D. Stoffer, 1989: Chaos in almost periodic systems. Z. Angew. Math. Phys., 40, 592-602.

Poje, A. C., and G. Haller, 1999: Geometry of cross-stream mixing in a double-gyre ocean model. J. Phys. Oceanogr., 29, 16491665

Poulain, P.-M., 1999: Drifter observations of surface circulation in the Adriatic Sea between December 1994 and March 1996. J. Mar. Syst., 20, 231-253.

_ 2001: Adriatic Sea surface circulation as derived from drifter data between 1990 and 1999. J. Mar. Syst., 29, 3-32.

Rogerson, A., P. D. Miller, L. J. Pratt, and C. K. R. T. Jones, 1999: Lagrangian motion and fluid exchange in a barotropic meandering jet. J. Phys. Oceanogr., 29, 2635-2655.

Rom-Kedar, V., and S. Wiggins, 1990: Transport in twodimensional maps. Arch. Ration. Mech. Anal., 109, 239-298.

— A. Leonard, and S. Wiggins, 1990: An analytical study of transport, mixing, and chaos in an unsteady vortical flow. $J$. Fluid Mech., 214, 347-394.

Rüssmann, H., 1989: Non-degeneracy in the perturbation theory of integrable dynamical systems. Number Theory and Dynamical Systems, M. M. Dodson and J. A. G. Vickers, Eds., London Mathematical Society Lecture Notes Series, Vol. 134, Cambridge University, 5-18.
Rypina, I. I., M. G. Brown, F. J. Beron-Vera, H. Koçak, M. J. Olascoaga, and I. A. Udovydchenkov, 2007a: On the Lagrangian dynamics of atmospheric zonal jets and the permeability of the Stratospheric Polar Vortex. J. Atmos. Sci., 64, 3595-3610.

$\longrightarrow,-,-,-$, and $\longrightarrow, 2007 \mathrm{~b}$ : Robust transport barriers resulting from strong Kolmogorov-Arnold-Moser stability. Phys. Rev. Lett., 98, Abstract 104102, doi:10.1103/ PhysRevLett.98.104102.

Samelson, R. M., 1992: Fluid exchange across a meandering jet. $J$. Phys. Oceanogr., 22, 431-440.

_ - and S. Wiggins, 2006: Lagrangian Transport in Geophysical Jets and Waves: The Dynamical Systems Approach. Springer, 147 pp.

Sevryuk, M. B., 2007: Invariant tori in quasi-periodic non-autonomous dynamical systems via Herman's method. Discrete Contin. Dyn. Syst., 18, 569-595.

Shadden, S. C., F. Lekien, and J. E. Marsden, 2005: Definition and properties of Lagrangian coherent structures from finite-time Lyapunov exponents in two-dimensional aperidic flows. Physica D, 212, 271-304.

Ursella, L., P.-M. Poulain, and R. P. Signell, 2006: Surface drifter derived circulation in the northern and middle Adriatic Sea: Response to wind regime and season. J. Geophys. Res., 111, C03S04, doi:10.1029/2005JC003177.

Wiggins, S., 1992: Chaotic Transport in Dynamical Systems. Springer-Verlag, $301 \mathrm{pp}$.

—-1999: Chaos in the dynamics generated by sequences of maps, with applications to chaotic advection in flows with aperiodic time dependence. Z. Angew. Math. Phys., 50, 585616.

— 2005: The dynamical systems approach to Lagrangian transport in oceanic flows. Annu. Rev. Fluid Mech., 37, 295328.

Yi, Y., 1993: A generalized integral manifold theorem. J. Differ. Equations, 102, 153-187.

Yuan, G. C., L. J. Pratt, and C. K. R. T. Jones, 2004: Cross-jet Lagrangian transport and mixing in a $2 \frac{1}{2} 2$-layer model. J. Phys. Oceanogr., 34, 1991-2005.

Zaslavskii, G. M., and B. V. Chirikov, 1972: Stochastic instability of non-linear oscillations. Sov. Phys.-Usp., 14, 549-568. 